\title{
Treatment Options to Manage Wound Biofilm
}

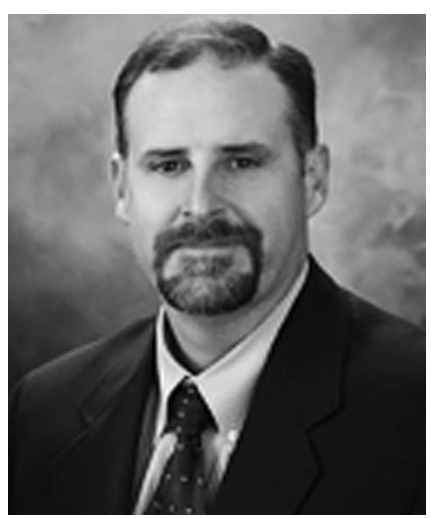

Curtis E. Jones

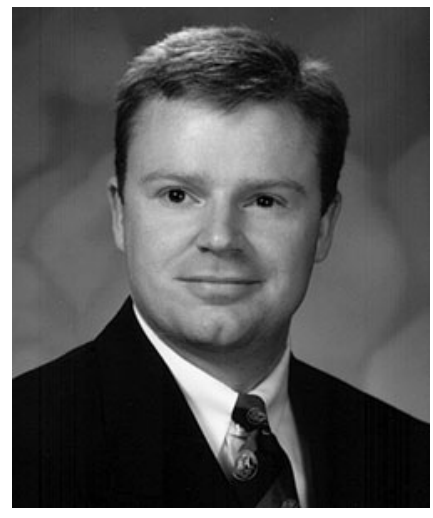

John P. Kennedy

Submitted for publication April 22, 2011

${ }^{*}$ Correspondence: Department of Pharmaceutical Sciences, South University School of Pharmacy, 709 Mall Blvd., Room 350, Savannah, GA 31406 (e-mail: jpkennedy@southuniversity .edu).

$$
\begin{aligned}
& \text { Abbreviations } \\
& \text { and Acronyms } \\
& A B F=\text { antibiofilm agents } \\
& A B X=\text { antibiotics } \\
& D F U=\text { diabetic foot ulcer } \\
& P U=\text { pressure ulcer } \\
& S X=\text { systemic (oral or } \\
& \text { intravenous) } \\
& T P L=\text { topical } \\
& V L U=\text { venous leg ulcer }
\end{aligned}
$$

\author{
Curtis E. Jones and John P. Kennedy* \\ Department of Pharmaceutical Sciences, South University School of Pharmacy, Savannah, Georgia.
}

Background: Bioburden is an accepted barrier to chronic wound healing. Defining the significance, phenotype, clinical classification, and treatment guidelines has been historically lacking of evidence and based on paradigms that do not represent the scientific or clinical reality.

The Problem: Chronic wound bioburden is typically abundant, polymicrobial, and extremely diverse. These microbes naturally adopt biofilm phenotypes, which are quite often viable but not culturable, thereby going undetected. The failures of culture-based detection have led to abandonment of routine bioburden evaluation and aggressive treatment or, worse, to assume bioburden is not a significant barrier. Predictably, treatment regimens to address biofilm phenotypes lagged behind our diagnostic tools and understanding.

Basic/Clinical Science Advances: Microbial DNA-based diagnostic tools and treatment regimens have emerged, which provide and leverage objective information, resulting in a dramatic impact on outcomes.

Relevance to Clinical Care: Modern medicine demands decisions based on objective evidence. The diagnostic and treatment protocols reviewed herein empower clinicians to practice modern medicine with regard to bioburden, with DNA level certainty.

Conclusion: Bioburden is a significant barrier to healing for all chronic wounds. Molecular diagnostics provide the first objective means of assessing wound bioburden. The accuracy and comprehensive data from such diagnostic methodologies provide clinicians with the ability to employ patient-specific treatment options, targeted to each patient's microbial wound census. Based on current outcomes data, the most effective therapeutic options are topical (TPL) antibiofilm agents (ABF) combined with TPL antibiotics (ABX). In specific patients, systemic $\mathrm{ABX}$ and selective biocides are also appropriate, but not exclusive of $\mathrm{ABF}$ combined with TPL ABX.

\section{BACKGROUND}

CONTINUING RESEARCH IN THE AREA of wound care indicates that bacterial bioburden and biofilm represents a universal barrier to healing. The presence of biofilm can be pronounced or imperceptible to the naked eye depending on the bacterial census and individual patient factors. The primary and most effective treatment of biofilm infections is physical removal followed by inhibition of reconstitution with antibiofilm agents $(\mathrm{ABF})$, antibiotics
(ABX), and selective biocides. However, a unified diagnosis of infection remains unsettled.

The use of topical (TPL) antimicrobials, $\mathrm{ABX}$, and biocides in wound care is ubiquitous. Historically, modern TPL therapy began with the treatment of superficial wounds following the discovery and commercial production of bacitracin, neomycin, and polymyxin B. During the last 50 years, the majority of $\mathrm{ABX}$ in use today were developed and first marketed-many for TPL use. 
However, wound care lacks consensus as to the appropriate utilization frequency and clinical application. ${ }^{1}$ This lack of consensus primarily resides with the absence of controlled clinical trials in chronic wounds supporting the efficacy of TPL ABX, as well as persistent suggestions that the use of TPL ABX subjects patients to increased rates of sensitization and antibiotic resistance.

\section{TARGET ARTICLES}

1. Wolcott RD, Cox SB, and Dowd SE: Healing and healing rates of chronic wounds in the age of molecular pathogen diagnostics. J Wound Care 2010; 7: 272.

2. Dowd SE, Wolcott RD, Jones CE, and Kennedy JP: Molecular bioburden diagnostics \& personalized medicine in wound care: theranostic outcomes assessment. J Wound Care 2011; 5: 232.

3. Percival SL, Hill KE, Malic S, Thomas DW, and Williams DW: Antimicrobial tolerance and the significance of persister cells in recalcitrant chronic wound biofilms. Wound Rep Reg 2011; 19: 1 .

\section{CLINICAL PROBLEM ADDRESSED}

Controversy surrounds the definition of "infection" within the field of chronic wounds. ${ }^{2}$ The field lacks consensus regarding the impact of microbial bioburden or biofilm within chronic wounds. References suggest that the presence of bacteria in a wound, within itself, is not indicative of infection. ${ }^{3}$ The lack of definitive consensus and subjective designations such as contamination, colonization, critical colonization, and infection are largely academic designations without objective measures requiring multiple, conditional variables assessed largely on a visual basis, to define the impact or presence of bioburden and biofilm. However, quantification of bacteria would appear pivotal in this consideration. The simple fact remains that under normal conditions, subcutaneous structures have no bacteria in residence. Logic would dictate that maintenance of viable tissue as closely as possible to an unimpaired condition would promote the return of normal physiology and function. Hence, the persistent inability to definitively and objectively identify, quantify, and diagnose the impact of bioburden hinders the standardization of treatment.

The use of TPL agents to control bioburden is a universal but largely subjective practice. A wide variety of xenobiotics are utilized to reduce bio- burden with the intention of creating an environment conducive to closure. Silver, iodine, silver sulfadiazine, mupirocin, and even medicinal honey are extensively utilized within the field. Systemic (SX) antibiotic usage in this population is also considerable. In addition to concurrent disease states, the literature reports that approximately $60 \%-66 \%$ of these patients receive antibiotic therapy associated with treatment of their wounds. ${ }^{4,5}$ However, the same routinely utilized ABX are summarily dismissed for TPL delivery without clinical evidence or justification. Despite the frequency of SX antibiotic use as well as the lack of contextual evidence, antibiotic resistance is often suggested as rationale to avoid TPL antibiotic therapy in wound care. In opposition to these suggested cautions, other medical disciplines continue to promote and provide evidence that TPL therapy minimizes the opportunity for resistance. By way of example, a polymyxin B study of 250 aural isolates of Pseudomonas aeruginosa noted for resistance development resulted in only one strain $(0.4 \%)$ reported as intermediately sensitive, whereas no strains were resistant, despite common TPL use for over 30 years. ${ }^{6-8}$ Therefore, the clinical problem may reside less in the lack of direct clinical evidence than the field's ability to appreciate evidence displayed within parallel disciplines in medicine. In alignment, the significant advantages of combining new $\mathrm{ABF}$ and/or biocides in combination with TPL ABX for chronic wound therapy are also discouraged de facto and without evidence, whereas other disciplines adopt these combinations for their merits.

\section{RELEVANT BASIC SCIENCE CONTEXT}

Expanding and maturing scientific evidence suggests that the acknowledgment of polymicrobial bacterial biofilm phenotypes in conjunction with the extensive bacterial diversity, which has been recently elucidated within chronic wounds, far surpasses the practical accuracy and economic feasibility of traditional culture methods. ${ }^{9}$ With the application of recently advanced DNA-based diagnostic methods (molecular methods), expanding diversity has been displayed through the relatively unlimited species identified to date, as well the heretofore unrealized ability to objectively quantify the relative bacterial census within a chronic wound. ${ }^{9}$ The evolution and application of this technology now forces scientists and clinicians alike to reevaluate the paradigm of the diagnosis of "infection" and consider new methods of therapy for patients that can leverage this acknowledgment.

The classic "infection continuum" has been described as contamination, followed by colonization, 
critical colonization, and finally, outright "infection." However, this continuum does not reflect recent advances in our understanding of the microbia/ host interfacial relationship. A more current description paraphrased for the reader might be contamination, attachment, proliferation, and critical concentration (of signaling molecules rather than bacterial counts), followed by the subsequent natural adoption of a biofilm phenotype, which becomes progressively polymicrobial given opportunity.

Regardless of medical discipline, current treatment guidelines based upon this updated paradigm, where they exist, all ideally employ the same strategies including physical disruption or excision, $\mathrm{ABF}$, high concentration $\mathrm{ABX}$, and biocides. ${ }^{10-12}$ A critical advantage for wound care clinicians is ready access to the affected site, which allows the employment of all available strategies, wherein other tissues impacted by biofilm infections are more limited (e.g., endocarditis).

For the purpose of this chapter, only "selective" biocides (biocides that are more cidal to microbes than the host cells) are relevant when biocides are cited. Such biocides include silver and iodine derivatives as well as methylene blue-gentian violet combinations. Alternatively, biocides that are not selective are not relevant within the context of the discussion herein, by way of example, Dakin's solution and hydrogen peroxide are expressly excluded. ABF are defined herein as agents that impart defensive biofilm controls including inhibition of microbial attachment, inhibition of planktonic conversion to biofilm phenotypes, and/or stimulation of biofilm phenotype reversion back to planktonic. $\mathrm{ABF}$, as described within, do not include ABX, which do impact biofilm phenotypes, but are considered offensive agents classified independently of antibiofilm compounds.

\section{EXPERIMENTAL MODEL OR MATERIAL: ADVANTAGES AND LIMITATIONS}

Molecular diagnostics are available to provide comprehensive, rapid, and accurate microbial detection and quantification of previously unidentifiable organisms, including yeast and fungi. However, although major resistance factors are also elucidated, classical species susceptibility is not provided. That said, one must consider the value of classic susceptibility testing when performed on species that actually do not represent the dominant or comprehensive microbial census. Further, traditional susceptibility testing continues to be based upon planktonic phenotypes, at concentrations achievable by SX delivery, whereas the evidence reports biofilm phenotype resistance levels 100 - to 1,500 -fold greater than planktonic susceptibilities. ${ }^{10,13}$

Fortunately, for wound care, the concentrations and combination therapies achievable by TPL delivery address these limitations as they have in other medical disciplines that rely upon and promote TPL delivery. ${ }^{14,15}$ As a notable limitation, research and identification of $\mathrm{ABF}$ are in their infancy, limiting the full utility of the promise they provide. Additionally, perhaps the "dirty secret" of DNA guided personalized medicine remains that truly patient specific therapies will not produce commercial volumes to justify mass-production. This will likely necessitate a return to specialized pharmacy practices, trained and skilled in the custom preparation of such patient-specific medications.

\section{DISCUSSION OF FINDINGS AND RELEVANT LITERATURE}

With the advent of molecular methods, it is apparent that the diversity of bacterial species involved in wound infection and the presence of the biofilm phenotype has been underestimated with traditional microbiological methods. ${ }^{16}$ Therefore, it should be anticipated that utilization of an increased number of antimicrobial and antibiotic agents will be necessary to adequately address the true censes in nonhealing wounds. Wolcott et al. was able to increase complete closure rates from $48 \%$ to $62 \%$ over a 6 -month study period utilizing multiple concurrent strategies including frequent debridement and molecular diagnostics to guide SX antibiotic intervention. ${ }^{17}$ As a result, SX antibiotic usage increased from $32 \%$ to $67 \%$ of patients receiving therapy. Although there was an overall increase in antibiotic usage, the increased utilization remains in alignment with the literature for this patient population. ${ }^{4,5}$

Treatment of biofilm phenotypes presents specific challenges related to structural and metabolic modifications exhibited by bacteria. ${ }^{18}$ The presence of extracellular polymeric substance and reduced metabolic activity within the biofilm presents a physical and mechanistic barrier to the effectiveness of traditional $\mathrm{ABX}$, which generally target metabolic and synthetic processes of bacteria. Antimicrobial concentrations required for inhibitory activity against biofilm phenotypes may be several orders of magnitude higher than for the planktonic phenotype. ${ }^{10,13}$ While appreciating the degraded vasculature of wound tissues, the ability to deliver SX concentrations in alignment with this evidence, for sufficient durations, and without host toxicity are progressively remote.

However, the use of TPL agents and therapy in the field of wound care is commonplace. TPL 
delivery offers distinct advantages including ready access to the desired site, the ability to deliver significantly higher local drug concentrations (i.e., increased therapeutic efficacy and decreased SX exposure/toxicity), the ability for individualized treatment options (i.e., multiple therapeutics simultaneously), as well as the ability to provide far more economical therapy.

The controversy regarding TPL antibiotic therapy is somewhat surprising, given that other specialties within medicine recognize and embrace TPL therapy, regularly citing TPL ABX as superior to SX options. ${ }^{14,15}$ The disciplines of dentistry, dermatology, ophthalmology, orthopedics, pulmonology, gynecology, and otolaryngology utilize TPL and localized drug therapy for these merits. In recent years, the utilization of inhaled medications and ABX for therapy and maintenance of cystic fibrosis has experienced significant consideration and utilization. ${ }^{19}$ The field of otolaryngology has perhaps embraced the use of TPL ABX to the greatest extent of any discipline with the present consideration of TPL ABX to be a superior treatment over SX agents in conditions of chronic infection, such as otitis externa and persistent otitis media. ${ }^{14,15}$ Given the ready access to the site of application, the analogous chronic states of bacterial presence, the acknowledgment of biofilm phenotypes, and the inclusion of these therapies in the standard of care within parallel medical disciplines, the use and consideration of TPL antibiotic therapy in the discipline of wound care should be reconsidered.

As evidence in this regard, Dowd et al. reported significantly increased rates of wound closure with the multiple concurrent strategies of biofilm-based wound care and the combination of individualized TPL therapy guided by molecular diagnostics. ${ }^{17}$ This analysis included over 1,300 patients receiving treatment guided by traditional culture (Control), SX ABX guided by molecular diagnostics (TG1), or TPL ABF/ABX combination therapy guided by molecular diagnostics (TG2). The healing rate was significantly increased with the use of molecular diagnostics and TPL therapy $($ Control $=48 \%$; TG1 $=62 \%$;

\section{TAKE-HOME MESSAGE}

\section{Basic science advances}

- DNA-based molecular methods allow objective species determination and overall and relative quantification. These methods are not subject to cultivation biases so evident with traditional culture. Molecular methods are also available that are comprehensive for bacteria, yeast, and fungi, thereby providing the fully elucidated microbial reality within wound samples that previously eluded researchers and clinicians. The application of DNA-based molecular methods represents the next step in the ability to determine critical concentrations of bacterial presence and to develop an objective diagnosis of infection.

- ABF have been previously recognized as effective interventions to improve patient outcomes. Recent research in the field has further demonstrated their synergy with antibiotic therapy.

- TPL drug delivery research and practice has advanced to include sustained release durations, pivotal to extending the effectiveness of $A B F$ and $A B X$ alike.

\section{Clinical science advances}

- Bioburden is a significant barrier to healing for all chronic wounds, regardless of etiology, and is the only barrier in which clinicians have full control to manage. As bioburden is universal, it can be a universal treatment strategy for all chronic wounds.

- Diagnostic tools with DNA level certainty can empower a specific and targeted management strategy to effectively control the contribution of bioburden as a barrier to healing.

- Any strict recommendation within the wound care discipline against the use of TPL ABX in wound care is unsupported by evidence and unfounded with the consideration of accepted evidence to the contrary in parallel disciplines of medicine addressing chronic infections (e.g., dentistry, dermatology, pulmonology, and otolaryngology).

- Recent wound care evidence for TPL antibiotic therapy as well as prudent application of evidence from parallel disciplines of medicine offers new options for progressive wound therapy, further supported by current wound evidence. TPL ABX in appropriate combinations are also theoretically and practically sound strategies for targeting biofilm phenotypes.

- Wound clinicians enjoy a significant advantage for managing biofilm over many other disciplines plagued by chronic infection. Namely, physical access to the affected tissues must be leveraged fully to optimize patient outcomes through physical disruption via regular, if not weekly, debridement and application of TPL ABF in combination with ABX.

- In addition, given the physiologic focus of the field, the authors encourage the discipline of wound care to not only consider the current evidence but also further challenge the efficacies reported herein within their own practice as a part of any such consideration. Any objective review must also include the evidence and accepted best practices of parallel disciplines in medicine, particularly with respect to the significant advantages of TPL therapeutics and ABX.

\section{Relevance to clinical care}

- Given the present innovation occurring within the field of DNA-based molecular diagnostic methods, we propose that the debated definition of "infection" in wounds will ultimately be reduced to objective and quantifiable measures currently lacking. Moreover, these diagnostic tools 
TG2 $=90 \%$ ). In alignment, the reduction in median days to healing was quite significant $\quad($ Control $=177 ; \quad$ TG1 $=77$ $[p<0.001]$; TG2 = $28 \quad[p<0.001])$. Further, statistical significance was demonstrated for both healing rate and days to healing in all subgroup analyses (diabetic foot ulcer, venous leg ulcer, pressure ulcer, and nonhealing surgical). When successive percentages of healed patients within each group were compared, patients receiving topically delivered $\mathrm{ABF} /$ ABX combinations healed two- to fourfold faster than Control.

In the present study, Wolcott et al. utilized ABX that are commonly used in TPL and SX therapy in wound care (i.e., clindamycin, metronidazole, amikacin, etc.). However, the treatment deviated from the predominant practice of a single active agent per treatment. By the compounding of individualized treatment gels, the diverse wound census was more comprehensively addressed, and combinations with ABF could be leveraged to potentiate the antibiotic regimen. ${ }^{20}$ Despite averaging about three $\mathrm{ABX}$ per patient, each ounce of TPL therapy contained a fraction of a single SX dose.

\section{INNOVATION}

The present innovation in wound care can be attributed to the development of accurate and quanti- allow clinicians to comprehensively and quantitatively access bioburden, regardless of phenotype, with DNA level certainty.

- Subjective opinion and paradigms aside, the improved outcomes disclosed herein are not biased by academic arguments regarding the relevance of microbial phenotypes (planktonic vs. biofilm), clinical arguments for determinations of or definitions for "infection," or subjective arguments regarding TPL therapeutics and ABX. Rather, the current evidence reviewed in this chapter strongly supports that bioburden is a significant barrier for all chronic wounds, regardless of etiology or clinical signs of infection. Further, the aggressive management of chronic wound burden significantly improves patient outcomes, most efficaciously via TPL drug delivery. tative molecular diagnostic methods for the determination of bacterial species, advances in the identification and delivery of ABF, as well as an ever more appreciated addition of biofilm phenotypes to any description of an infection continuum. With this powerful, objective data, clinicians are empowered to make informed decisions regarding the scope, direction, and efficacy of bioburden treatment. In addition, the development of individualized TPL therapies, utilizing $\mathrm{ABF}$ and overlapping or synergistic antibiotic combinations in alignment with aggressive SX regimens, provide flexible and comprehensive adjuncts to the basic tenets of biofilm-based wound care.

\section{SUMMARY ILLUSTRATIONS}

A facilitative illustration of the goals of biofilm management is shown below. Simply stated, those

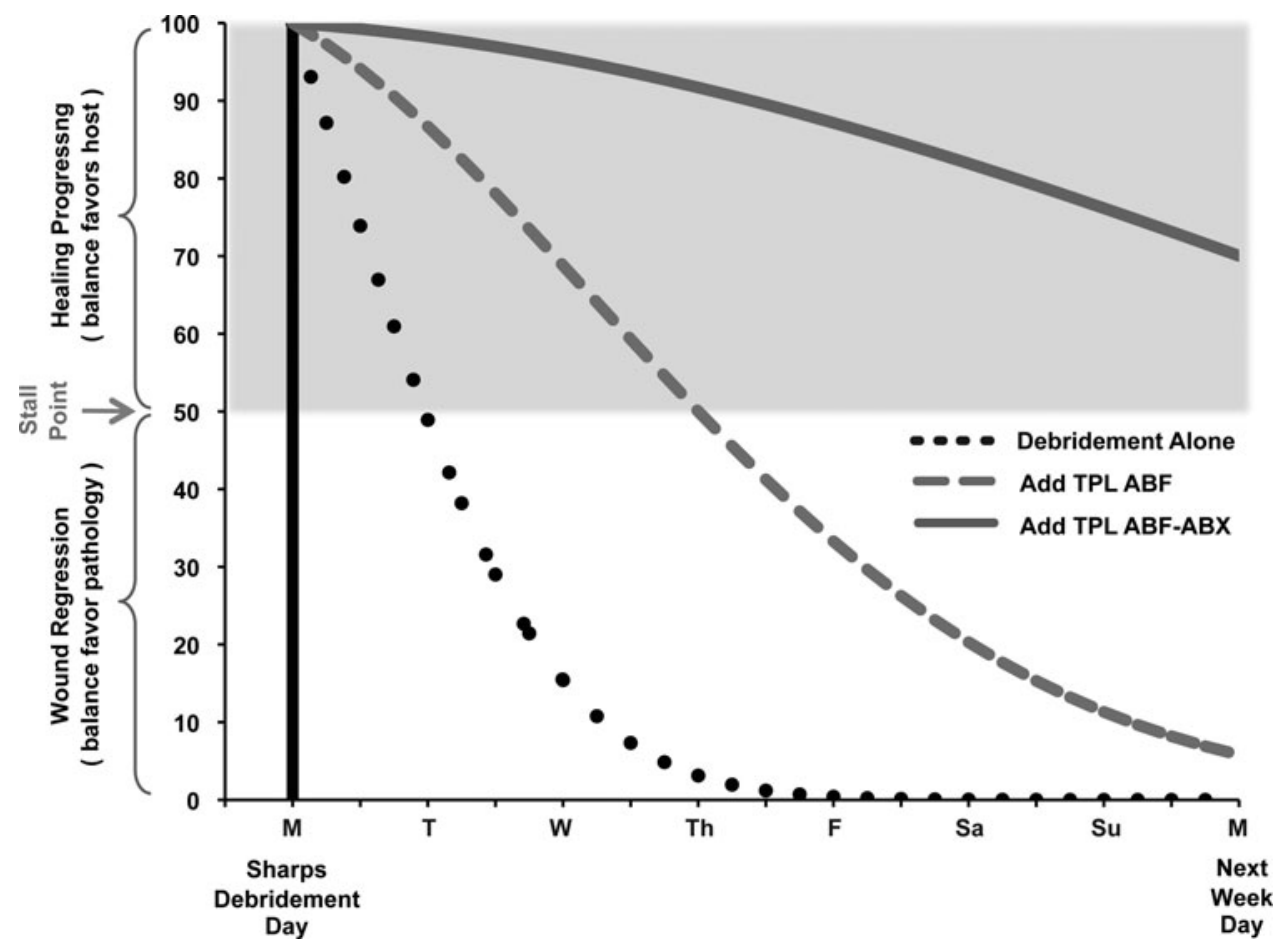




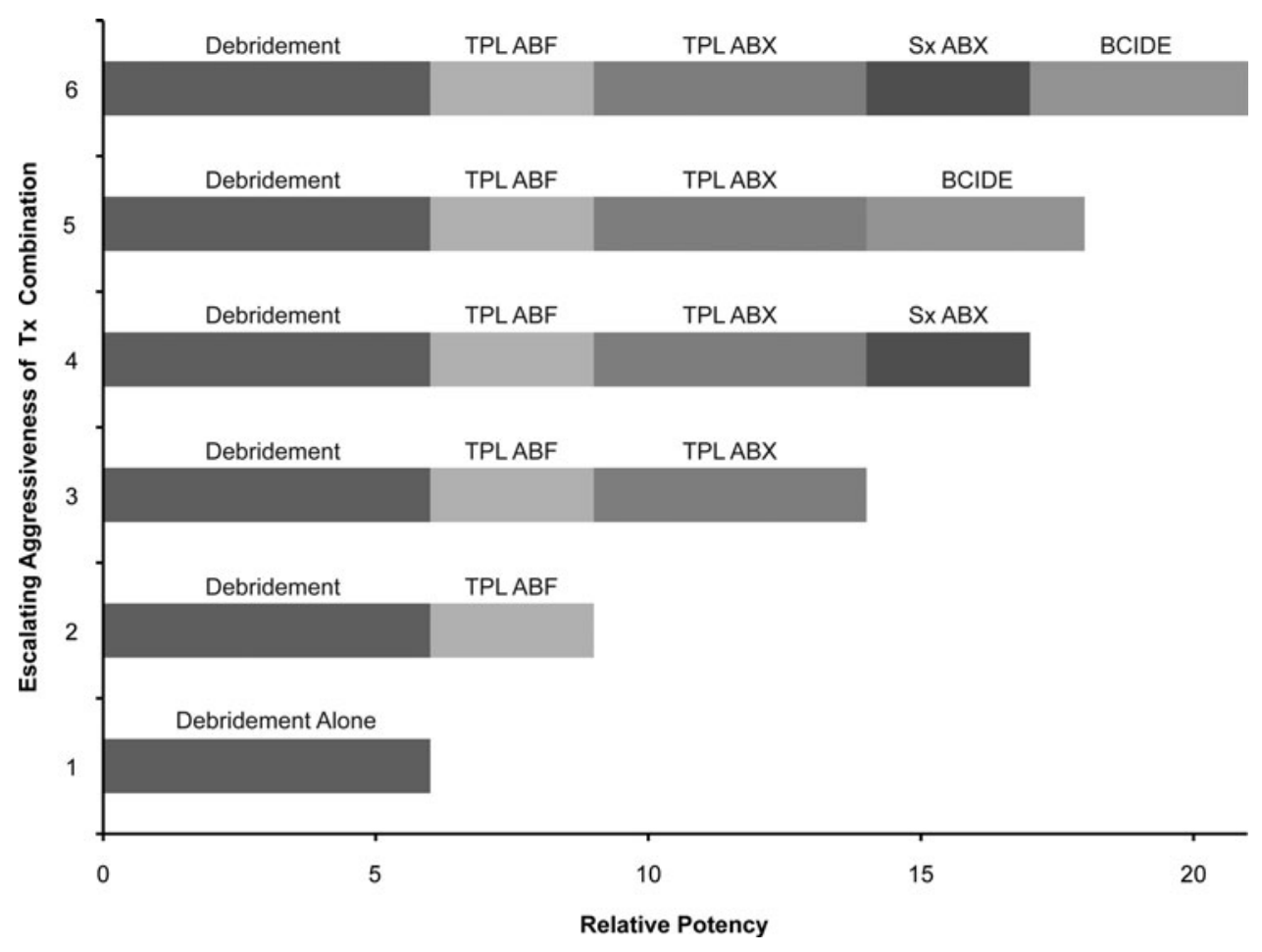

goals are to (1) apply therapeutic interventions that reduce this bioburden, thereby tipping the healing balance in favor of the host, and (2) to prolong the duration the balance remains favorable to healing. In this illustration, the initial intervention is debridement, which is foundational for biofilm management across all medical disciplines. The subsequent two biofilm trajectories illustrate the advantages and relative potencies of debridement supplementation with TPL ABF alone or with $\mathrm{TPL} \mathrm{ABF}+\mathrm{ABX}$ in combination.

The relative potencies of biofilm management options available to clinicians are depicted above. For simplicity, evident synergies of the combinations are not considered..$^{10-12,20}$ Following foundational debridement, progressively escalating levels of interventional options are illustrated. Weekly debridement and TPL ABF combined with TPL $\mathrm{ABX}$ were the primary interventional levels employed in the target study cited in this chapter. ${ }^{17}$ SX ABX are appropriate when deep tissues (including bone and extended cellulitis) are involved or anytime a limb is in jeopardy of amputation, but not at the exclusion of TPL ABF/ABX combinations. Similarly, biocide additions offer clinical value, but are less selective (more toxic) and less targeted. Once under control, the authors' recommended prudent order for discontinuance (de-escalation) is biocides, followed by SX ABX, and optionally all TPL interventions when wound closure is imminent.

\section{CAUTION, CRITICAL REMARKS, AND RECOMMENDATIONS}

Frequent debridement is foundational for biofilm management. The authors recommend weekly sharp debridement until the wound becomes selfsustaining upon approaching closure. The target efficacy study cited herein supports molecular diagnostics and bioburden treatment for all chronic wounds, regardless of clinical signs of infection. TPL combinations of $\mathrm{ABF}+\mathrm{ABX}$ are also recommended as baseline treatments, as significantly greater healing rates and shortened days to healing were returned from this treatment group.

During such treatment regimens, caution should be applied if progressing wounds ultimately stall. This should not be misinterpreted as microbial resistance. In practice, resistance has not been noted under these protocols in 3 years of utility. Rather, a stalled wound is an indication that the microbial census has shifted to alternative species, requiring rediagnosis and subsequent modification of the treating agents. It has also identified noncompliance in a few patients.

Chronic wounds require multiple concurrent strategies, which the authors fervently support. However, bioburden management is understood as synergistic to all. By way of example, the use of dermal substitutes is significantly augmented by aggressive management of bioburden prior to and during the employment of these valued interventions. Within our own clinics, and those that we 
consult with, the combination has significantly impacted the rates of success within the reimbursement limitations imposed on such substitutes.

\section{FUTURE DEVELOPMENT OF INTEREST}

Although the evidence demonstrated by biofilm control measures in the current literature is a cause for great enthusiasm, these paradigms and strategies will logically continue to mature into ever more efficacious protocols. Relative to diagnostics, logical areas for future development include the addition of inflammatory markers to molecular analysis of bioburden and provisions for clinical access to biofilm phenotype susceptibility testing. From a treatment perspective, prudent focus of research and development resources include expanded screening and identification of
$\mathrm{ABF}$, investigations into the $\mathrm{SX}$ utility of $\mathrm{ABF}$, translation of the protocols highlighted herein into regimens for parallel disease states (e.g., prosthetic joint infections), as well as the elucidation of the role of steroids in chronic wounds.

\section{ACKNOWLEDGMENTS AND FUNDING SOURCES}

The authors have not received funding for this work.

\section{AUTHOR DISCLOSURE AND GHOSTWRITING}

C.E.J. and J.P.K. are clinical advisors for PathoGenius ${ }^{\circledR}$ Laboratories, a molecular diagnostics laboratory. Ghostwriters were not used in production of this article.

\section{REFERENCES}

1. Siddiqui $A R$ and Bernstein JM: Chronic wound infection: facts and controversies. Clinics in Derm 2010; 28: 519.

2. Lipsky BA and Hoey C: Topical antimicrobial therapy for treating chronic wounds. Clin Inf Dis 2009; 49: 1541

3. Bowler PG, Duerden BI, and Armstrong DG: Wound microbiology and associated approaches to wound management. Clin Microbio Reviews 2001; 14: 244.

4. Tammelin A, Lindholm C, and Hambraeus A: Chronic ulcers and antibiotic treatment. J Wound Care 1998; 7: 435.

5. Howell-Jones RS, Price PE, Howard AJ, and Thomas DW: Antibiotic prescribing for chronic skin wounds in primary care. Wound Repair Regen 2006; 14: 387.

6. Dohar JE, Kenna MA, and Wadowsky RM: In vitro susceptibility of aural isolates of Pseudomonas aeruginosa. Am J Otology 1996; 17: 207.

7. Klein JO: Strategies for decreasing multidrug antibiotic resistance: role of ototopical agents for treatment of middle ear infections. Am J Managed Care 2002; 8 (Suppl 14): 345

8. Weber PC, Roland PS, Hannley M, Friedman R, Manolidis S, Matz G, Owens F, Rybak L, and Stewart MG: The development of antibiotic resistant organisms with the use of ototopical medications. Otolaryngol Head Neck Surg 2004; 130 (Suppl 3): 89

9. Dowd SE, Sun Y, Secor PR, Rhodes DD, Wolcott BM, James GA, and Wolcott RD: Survey of bacterial diversity in chronic wounds using Pyrosequencing, DGGE, and full ribosome shotgun sequencing. BMC Microbiol 2008; 8: 43.

10. El-Azizi M, Kanchanapoom T, and Khardori N: In vitro activity of vancomycin, quinupristin/dalfopristin, and linezolid against intact and disrupted biofilms of staphylococci. Ann Clin Microb Antimicrob 2005; 4: 2

11. Wolcott R, Rumbaugh K, James G, Schultz G, Phillips $P$, Yang 0 , Watters $C$, Stewart $P$, and Dowd S: J Wound Care 2010; 19: 8, 320.

12. Wolcott R and Rhoads D: J Wound Care 2008; 17: 4, 272

13. Hill KE, Malic S, McKee R, Rennison T, Harding KG, Williams DW, and Thomad DW: An in vitro model of chronic wound biofilms to test wound dressings and assess antimicrobial susceptibilities. J Antimicrob Chemother 2010; 65: 1195

14. Macfadyen CA, Acui JM, and Gamble C: Systemic antibiotics versus topical treatments for chronically discharging ears with underlying eardrum perforations. Cochrane Database Syst Rev 2006; Issue 1: Art No.: CD005608.
15. Dohar J, Giles W, Roland P, Bikhazi N, Carroll S, Moe R, Reese B, Dupre S, Wall M, Stroman D, McLean C, and Crenshaw K: Topical Ciprofloxacin/ Dexamethasone superior to oral amoxicillin/ clavulanic acid in acute otitis media with otorrhea through tympanostomy tubes. Pediatrics 2006; 118: 561

16. Wolcott RD, Cox SB, and Dowd SE: Healing and healing rates of chronic wounds in the age of molecular pathogen diagnostics. J Wound Care 2010; 7: 272

17. Dowd SE, Wolcott RD, Wolcott RD, Jones CE, and Kennedy JP: Molecular bioburden diagnostics \& personalized medicine in wound care: Theranostic outcomes assessment. J Wound Care 2011; 5: 232.

18. Percival SL, Hill KE, Malic S, Thomas DW, and Williams DW: Antimicrobial tolerance and the significance of persister cells in recalcitrant chronic wound biofilms. Wound Rep Regeneration 2011; 19: 1.

19. Heijerman H, Westerman E, Conway S, Touw D, and Doring G: Inhaled medication and inhalation devices for lung disease in patients with cystic fibrosis: a European consensus. J Cystic Fibrosis 2009; 9: 295

20. Estrela A and Abraham WF: Combining biofilmcontrolling compounds and antibiotics a promising new way to control biofilm infections. Pharmaceuticals 2010; 3: 1374 